\title{
Local aging phenomena close to magnetic surfaces
}

\author{
Florian Baumann ${ }^{1,2}$ and Michel Pleimling ${ }^{3}$ \\ ${ }^{1}$ Laboratoire de Physique des Matériaux (CNRS Unité Mixte de Recherche 7556), Université Henri Poincaré Nancy I, \\ Boîte Postal 239, F-54506 Vandouvre lès Nancy Cedex, France \\ ${ }^{2}$ Institut für Theoretische Physik I, Universität Erlangen-Nürnberg, Staudtstraße 7B3, D-91058 Erlangen, Germany \\ ${ }^{3}$ Department of Physics, Virginia Polytechnic Institute and State University, Blacksburg, Virginia 24061-0435, USA
}

(Received 18 April 2007; revised manuscript received 19 June 2007; published 20 September 2007)

\begin{abstract}
Surface aging phenomena are discussed for semi-infinite systems prepared in a fully disordered initial state and then quenched to or below the critical point. In addition to solving exactly the semi-infinite Ising model in the limit of large dimensions, we also present results of an extensive numerical study of the nonequilibrium dynamical behavior of the two-dimensional semi-infinite Ising model undergoing coarsening. The studied models reveal a simple aging behavior where some of the nonequilibrium surface exponents take on values that differ from their bulk counterparts. For the two-dimensional semi-infinite Ising model we find that the exponent $b_{1}$ that describes the scaling behavior of the surface autocorrelation vanishes. These simulations also reveal the existence of strong finite-time corrections that to some extent mask the leading scaling behavior of the studied two-time quantities.
\end{abstract}

DOI: 10.1103/PhysRevB.76.104422

PACS number(s): 05.70.Np, 75.40.Gb, 75.70.Rf, 05.50.+q

\section{INTRODUCTION}

Intriguing phenomena are observed when bringing simple ferromagnets out of equilibrium through a temperature quench. ${ }^{1-5}$ Consider a ferromagnetic system prepared at high temperatures, i.e., in a disordered and uncorrelated initial state, that is suddenly quenched onto or below the critical point. If the quench is onto the critical point, critical dynamics sets in, yielding a dynamical correlation length that increases with time. For a quench inside the ordered phase, the formation and growth of well-ordered domains are observed. Interestingly, these two physically very different cases have in common that the relevant length scale $L$ (which in the first case is the dynamical correlation length, whereas in the second case it is given by the typical extent of the ordered domains) increases as a simple power law of time

$$
L(t) \sim t^{1 / z}
$$

Assuming nonconserved dynamics (which is the only dynamics studied in this paper) one finds for the dynamical exponent $z$ the value 2 below the critical temperature $T_{c}$, whereas at $T_{c}$ the dynamical exponent may take on values slightly larger than 2 .

The power-law growth (1) is responsible for many nonequilibrium phenomena which are usually summarized under the header of simple aging. Thus, it follows directly from Eq. (1) that two-time quantities, such as dynamical correlation and response functions, display dynamical scaling. Introducing the time- and space-dependent order parameter $\phi(\vec{r} ; t)$, the correlation function can be written as

$$
C\left(t, s ; \vec{r}-\vec{r}^{\prime}\right)=\left\langle\phi(\vec{r} ; t) \phi\left(\vec{r}^{\prime} ; s\right)\right\rangle,
$$

whereas the response function, which measures the response of the order parameter at site $\vec{r}$ at time $t$ to an external field $h(\vec{r} ; s)$ acting on site $\vec{r}^{\prime}$ at time $s$, is defined by

$$
R\left(t, s ; \vec{r}-\vec{r}^{\prime}\right)=\left.\frac{\delta\langle\phi(\vec{r} ; t)\rangle}{\delta h\left(\vec{r}^{\prime} ; s\right)}\right|_{h=0} \quad(t>s) .
$$

In writing these equations we assume spatial translation invariance, as encountered in ferromagnetic bulk systems. The usually studied autocorrelation function $C(t, s)$ and autoresponse function $R(t, s)$ are obtained by setting $\vec{r}=\vec{r}^{\prime}$ in Eqs. (2) and (3), respectively. In the dynamical scaling regime with $t, s, t-s \gg \tau_{\text {micro }}$, where $\tau_{\text {micro }}$ is a microscopic time scale, these two-time quantities can be cast into a simple scaling form. For example, for the autocorrelation and the autoresponse functions we have

$$
C(t, s)=s^{-b} f_{C}(t / s) \text { and } R(t, s)=s^{-1-a} f_{R}(t / s),
$$

where $a$ and $b$ are nonequilibrium exponents, whereas $f_{C}$ and $f_{R}$ are scaling functions that for large arguments display a power-law decay

$$
f_{C}(y) \sim y^{-\lambda_{C} / z} \text { and } f_{R}(y) \sim y^{-\lambda_{R} / z},
$$

with the autocorrelation ${ }^{6,7}$ and the autoresponse exponents ${ }^{8}$ $\lambda_{C}$ and $\lambda_{R}$. At the critical point, the nonequilibrium exponents $a$ and $b$ can be expressed by known critical exponents, yielding $a=b=(d-2+\eta) / z$, where $d$ is the dimensionality of the system and $\eta$ is the usual static critical exponent governing the power-law decay of the spatial correlations at equilibrium. In the ordered phase one has $b=0$ and $a=1 / z$ for systems with exponentially decaying static correlations. ${ }^{1,9,10}$ At the critical point the autocorrelation exponent is related to the so-called initial slip exponent. ${ }^{11}$ In addition, autocorrelation and autoresponse exponents can be shown to be identical in systems with short-ranged initial correlations and purely relaxational dynamics. ${ }^{1,12}$

This briefly described simple aging scenario has been studied very intensively in bulk systems, but for systems bounded by surfaces the investigation of local aging processes close to surfaces is only at its very beginning., 33,14 The emerging interest in surface aging phenomena can be 
related to the increasing importance of surface dominated small systems in research and technology. Indeed, nonequilibrium processes are deeply affected by the presence of surfaces which can result in changes in the physical behavior even at macroscopic distances from the surface. In principle, these surface properties can be studied by x-ray scattering at grazing incidence.

Looking at critical systems bounded by surfaces, it is already well known that the static critical behavior at a surface is different from the bulk critical behavior, yielding new sets of static surface critical exponents. ${ }^{15-18}$ In fact, one even encounters different surface universality classes for a given bulk universality class, depending on the value of the surface couplings or on the existence of a surface field. Looking at the dynamics, it has to be noted that in the case of purely diffusive dynamics the dynamical exponent $z$ has the same universal value close to the surface as inside the bulk. ${ }^{19-21}$ One then expects a surface aging behavior similar to the bulk behavior, but with local nonequilibrium exponents and scaling functions of local two-time functions that differ from the corresponding bulk quantities.

In order to discuss the expected aging phenomenology close to a critical surface in more detail, let us consider an idealized semi-infinite lattice in $d$ dimensions where we write the position vector $\vec{r}$ as $\vec{r}=(\vec{x}, y)$. Here $\vec{x}$ is a $(d-1)$-dimensional vector parallel to the surface, whereas $y$ labels the layers perpendicular to the surface (with $y=1$ being the surface layer). With this we obtain the following generalizations for the correlation and response functions:

$$
\begin{gathered}
C\left(t, s ; y, y^{\prime}, \vec{x}-\vec{x}^{\prime}\right)=\left\langle\phi(\vec{x}, y ; t) \phi\left(\vec{x}^{\prime}, y^{\prime} ; s\right)\right\rangle, \\
R\left(t, s ; y, y^{\prime}, \vec{x}-\vec{x}^{\prime}\right)=\left.\frac{\delta\langle\phi(\vec{x}, y ; t)\rangle}{\delta h\left(\vec{x}^{\prime}, y^{\prime} ; s\right)}\right|_{h=0},
\end{gathered}
$$

where we assumed spatial translation invariance in the directions parallel to the surface. For $y, y^{\prime} \rightarrow \infty$ we recover the bulk quantities, whereas $y=y^{\prime}=1$ yields the surface correlation and response functions. Of special interest are the surface autocorrelation and autoresponse functions with $\vec{x}=\vec{x}^{\prime}$ that we write as $C_{1}(t, s):=C(t, s ; 1,1, \overrightarrow{0})$ and $R_{1}(t, s)$ $:=R(t, s ; 1,1, \overrightarrow{0})$. For these surface quantities, the simple scaling forms

$$
\begin{aligned}
& C_{1}(t, s)=s^{-b_{1}} f_{C_{1}}(t / s), \quad f_{C_{1}}(t / s) \sim(t / s)^{-\lambda_{C_{1}} / z}, \\
& R_{1}(t, s)=s^{-1-a_{1}} f_{R_{1}}(t / s), \quad f_{R_{1}}(t / s) \sim(t / s)^{-\lambda_{R_{1}} / z}
\end{aligned}
$$

are expected ${ }^{3,13}$ when $t, s$ and also the difference $t-s$ are large compared to some microscopic timescale. The scaling functions $f_{C_{1}}(t / s)$ and $f_{R_{1}}(t / s)$ should again display a simple power-law behavior for large values of $t / s$. General scaling arguments $^{3}$ allow us to express the surface exponents appearing in Eq. (6) through other known exponents

$$
a_{1}=b_{1}=\left(d-2+\eta_{\|}\right) / z \text { and } \lambda_{C_{1}}=\lambda_{R_{1}}=\lambda_{C}+\eta_{\|}-\eta,
$$

where $\eta_{\|}$is the static exponent that governs the decay of the correlations parallel to the surface. As for bulk systems, ${ }^{22,23}$ surface autocorrelation and autoresponse functions can be combined to yield the surface fluctuation-dissipation ratio ${ }^{13}$

$$
X_{1}(t, s)=\frac{T_{c} R_{1}(t, s)}{\partial_{s} C_{1}(t, s)},
$$

with a universal limit value

$$
X_{1}^{\infty}=\lim _{s \rightarrow \infty}\left[\lim _{t \rightarrow \infty} X_{1}(t, s)\right]
$$

that characterizes the different dynamical surface universality classes. ${ }^{3}$ The scaling picture (6) and the relations between the various nonequilibrium exponents have been verified by one of us through a numerical study of the out-ofequilibrium dynamics of various critical semi-infinite Ising models. ${ }^{13}$ In addition, the critical semi-infinite Gaussian model $^{3}$ and the critical semi-infinite spherical model ${ }^{14}$ were also found to display this simple aging scenario.

Whereas at least some knowledge has accumulated in recent years on the local aging behavior close to critical surfaces, almost nothing is known on surface aging processes taking place in coarsening systems. In Ref. 14 we have looked at the out-of-equilibrium dynamical behavior of the semi-infinite spherical model. For this special model we have verified the existence of dynamical scaling and simple aging close to surfaces for quenches inside the ordered phase. Surprisingly, the nonequilibrium exponent $b_{1}$, describing the scaling behavior of the surface autocorrelation, was found to take on the value $b_{1}=1$, different from the standard value $b$ $=0$ of the corresponding exponent in bulk systems undergoing phase ordering. This result calls for a thorough investigation of surface aging phenomena in other semi-infinite systems with phase-ordering dynamics.

In this paper we continue our study of local aging processes in bounded ferromagnets. On the one hand, we discuss the semi-infinite short-range Ising model in the limit of high dimensions that can be solved exactly. On the other hand, we present results of extensive Monte Carlo simulations of the standard two-dimensional semi-infinite Ising model prepared at high temperatures and then quenched inside the ordered phase. These numerical results yield new and interesting insight into the local processes taking place in coarsening systems close to surfaces. All the systems studied have in common that the dynamical exponent takes on the value $z=2$.

The paper is organized as follows. In Sec II. we compute scaling functions and nonequilibrium exponents in the exactly solvable semi-infinite model. Our numerical results obtained from simulations of the two-dimensional semi-infinite Ising model undergoing phase ordering are then presented in Sec. III. Finally, in Sec. IV we draw our conclusions and summarize our results.

\section{QUENCHING SEMI-INFINITE SYSTEMS FROM HIGH TEMPERATURES: EXACT RESULTS}

Exactly solvable models are often quite unrealistic and even artificial. One of the reasons for nevertheless studying this kind of models in physics is to obtain a guidance for the development of a future more sophisticated theoretical ap- 
proach. With this in mind, we discuss in the following the nonequilibrium dynamical behavior of the exactly solvable short-range semi-infinite Ising model in the limit of high dimensions that is prepared in an uncorrelated initial state with vanishing magnetization and then quenched below or at the critical point. As in that limit the model is mean-fieldlike, we expect the same critical exponents and the same scaling functions (up to some numerical prefactors) as those found in the semi-infinite Gaussian model. ${ }^{3}$

The out-of-equilibrium behavior of the bulk Ising model with nearest neighbor ferromagnetic interactions has recently been studied in the limit of a large number $d$ of space dimensions. ${ }^{24}$ Here we generalize the calculations of Garriga et al. to the semi-infinite case.

Using a semi-infinite hypercube with lattice constant 1 , the Hamiltonian of our model can be written in the very general form

$$
\begin{aligned}
\mathcal{H}= & -\frac{J_{s}}{2 d} \sum_{\left(\vec{x}, \vec{x}^{\prime}\right)} \sigma_{\vec{x}, 1} \sigma_{\vec{x}^{\prime}, 1}-\frac{J_{b}}{2 d} \sum_{y \geqslant 2} \sum_{\left(\vec{x}, \vec{x}^{\prime}\right)} \sigma_{\vec{x}, y} \sigma_{\vec{x}^{\prime}, y} \\
& -\frac{J_{b}}{2 d} \sum_{y \geqslant 1} \sum_{\vec{x}} \sigma_{\vec{x}, y} \sigma_{\vec{x}, y+1},
\end{aligned}
$$

where the sum over $\left(\vec{x}, \vec{x}^{\prime}\right)$ indicates a sum over all nearestneighbor pairs lying in the same layer. The spins can take on the values \pm 1 , and an additional field term can be added if needed. In writing Eq. (10) we take into account the layered structure of the lattice and distinguish between nearestneighbor pairs lying in a layer parallel to the surface and nearest-neighbor pairs belonging to different layers. As usual when dealing with semi-infinite systems, ${ }^{13}$ we have introduced a different coupling constant $J_{s}$ for interactions between nearest-neighbor spins located both in the surface layer. We will, however, restrict ourselves in the following to the special case $J_{s}=J_{b}=1$. On the one hand, this yields in the limit $d \rightarrow \infty$ the critical temperature $T_{c}=1$ (where we set $k_{B}$ $=1$ ), on the other hand we then encounter at the critical temperature the so-called ordinary transition ${ }^{13}$ where the bulk alone is critical.

The main difference between the present case and the model considered in Ref. 24 is of course the absence of spatial translation invariance in the direction perpendicular to the surface. Due to this, the time-dependent local fields that the spins experience are now layer dependent, leading to the expressions

$$
\begin{aligned}
h_{\vec{x}, y}(t)=h_{\vec{x}, y}^{\mathrm{ext}}(t)+ & \frac{1}{2 d}\left(\sigma_{\vec{x}, y+1}(t)+\sum_{\vec{x}^{\prime}(\vec{x})} \sigma_{\vec{x}^{\prime}, y}(t)\right) \quad \text { for } y=1, \\
h_{\vec{x}, y}(t)= & h_{\vec{x}, y}^{\mathrm{ext}}(t)+\frac{1}{2 d}\left(\sigma_{\vec{x}, y+1}(t)+\sigma_{\vec{x}, y-1}(t)\right. \\
& \left.+\sum_{\vec{x}^{\prime}(\vec{x})} \sigma_{\vec{x}^{\prime}, y}(t)\right) \quad \text { for } y \neq 1,
\end{aligned}
$$

where the sum over $\vec{x}^{\prime}(\vec{x})$ is the sum over the in-plane nearest-neighbor lattice sites $\vec{x}^{\prime}$ of $\vec{x}$. We added in these equations an external field $h_{\vec{x}, y}^{\text {ext }}(t)$ needed for the computation of the response function [for the computation of the correlation function, $h_{\vec{x}, y}^{\mathrm{ext}}(t)$ is, of course, set to zero]. Using heat-bath dynamics, these local fields $h_{\vec{x}, y}(t)$ (which depend on the dimension $d$ ) appear in the flip rates, as each spin will flip independently with the rate $\left\{1-\sigma_{\vec{x}, y}(t) \tanh \left[h_{\vec{x}, y}(t) / T\right]\right\} / 2$. It is important to note that in absence of an external magnetic field the magnetization remains at any time at is initial value zero everywhere in the sample.

\section{A. The correlation function}

In their paper ${ }^{24}$ Garriga et al. derived very general equations of motion for the one- and the two-time correlation functions $\mathcal{C}$ and $C$ that can also be used in our case by plugging in the layer-dependent local fields (11). Recalling that we still have invariance for spatial translations parallel to the surface, we can write the following equations:

$$
\begin{aligned}
\partial_{t} \mathcal{C}\left(t ; y, y^{\prime}, \vec{x}-\vec{x}^{\prime}\right)= & -2 \mathcal{C}\left(t ; y, y^{\prime}, \vec{x}-\vec{x}^{\prime}\right)+\left\langle\Delta \mathrm{t}_{\vec{x}, y}(t) \Delta \sigma_{\vec{x}^{\prime}, y^{\prime}}(t)\right\rangle \\
& +\left\langle\Delta \sigma_{\vec{x}, y}(t) \Delta \mathrm{t}_{\vec{x}^{\prime}, y^{\prime}}(t)\right\rangle, \\
\partial_{t} C\left(t, s ; y, y^{\prime}, \vec{x}-\vec{x}^{\prime}\right)= & -C\left(t, s ; y, y^{\prime}, \vec{x}-\vec{x}^{\prime}\right) \\
& +\left\langle\Delta \mathrm{t}_{\vec{x}, y}(t) \Delta \sigma_{\vec{x}^{\prime}, y^{\prime}}(s)\right\rangle
\end{aligned}
$$

where we use the notations $\Delta \mathrm{t}_{\vec{x}, y}(t):=\tanh \left[h_{\vec{x}, y}(t) / T\right]$ $-\left\langle\tanh \left[h_{\vec{x}, y}(t) / T\right]\right\rangle$ and $\Delta \sigma_{\vec{x}, y}(t)=\sigma_{\vec{x}, y}(t)-\left\langle\sigma_{\vec{x}, y}(t)\right\rangle$ for the deviations from the averages.

In the limit of large $d$ we can develop $\tanh \left[h_{\vec{x}, y}(t) / T\right]$ in $1 / d$ (recall that no external field is acting on the spins and that the local fields $h_{\vec{x}, y}$ are layer dependent) which then yields the following expressions for the equations of motion:

$$
\begin{aligned}
\partial_{t} \mathcal{C}\left(t ; y, y^{\prime}, \vec{x}\right)= & -2 \mathcal{C}\left(t ; y, y^{\prime}, \vec{x}\right)+\frac{\gamma}{2}\left(\mathcal{C}\left(t ; y+1, y^{\prime}, \vec{x}\right)\right. \\
& +\mathcal{C}\left(t ; y-1, y^{\prime}, \vec{x}\right)+\mathcal{C}\left(t ; y, y^{\prime}+1, \vec{x}\right) \\
& \left.+\mathcal{C}\left(t ; y, y^{\prime}-1, \vec{x}\right)+2 \sum_{\vec{z}(\vec{x})} \mathcal{C}\left(t ; y, y^{\prime}, \vec{z}\right)\right) \\
& +b\left(t ; y, y^{\prime}, \vec{x}\right) \\
\partial_{t} C\left(t, s ; y, y^{\prime}, \vec{x}\right)= & -C\left(t, s ; y, y^{\prime}, \vec{x}\right)+\frac{\gamma}{2}\left(C\left(t, s ; y+1, y^{\prime}, \vec{x}\right)\right. \\
& \left.+C\left(t, s ; y-1, y^{\prime}, \vec{x}\right)+\sum_{\vec{z}(\vec{x})} C\left(t, s ; y, y^{\prime}, \vec{z}\right)\right)
\end{aligned}
$$

with

$$
C\left(t, s ; 0, y^{\prime}, \vec{x}\right)=0=C(t, s ; y, 0, \vec{x}) .
$$

In writing these equations we exploit the spatial translation invariance parallel to the surface by setting $\vec{x}^{\prime}=\overrightarrow{0}$. The parameter $\gamma$ is given by $\gamma:=1 /(T d)$, whereas the sum over $\vec{z}(\vec{x})$ indicates a summation over the in-plane nearest-neighbor lattice sites of $\vec{x}$. The quantity $b\left(t ; y, y^{\prime}, \vec{x}\right)=\delta_{y, y^{\prime}} \delta_{\vec{x}, 0} \vec{b}(t ; y)$, which is needed to enforce the condition $\mathcal{C}(t ; y, y, \overrightarrow{0})=1$ for 
all times $t$, has to be determined self-consistently. ${ }^{24}$ In addition, the two-time correlator must yield the one-time correlator for $t=s$, i.e.,

$$
C\left(t, t ; y, y^{\prime}, \vec{x}\right)=\mathcal{C}\left(t ; y, y^{\prime}, \vec{x}\right)
$$

The solution of these equations of motion is outlined in the Appendix. For decorrelated initial conditions, our result is

$$
\begin{aligned}
C\left(t, s ; y, y^{\prime}, \vec{x}\right) \\
=e^{-(t+s)}\left\{I_{y-y^{\prime}}(\gamma(t+s))-I_{y+y^{\prime}}(\gamma(t+s))\right\} \\
\quad \times \prod_{i=1}^{d-1} I_{x_{i}}(\gamma(t+s))+\sum_{u \geqslant 1} \int_{0}^{s} d \tau \bar{b}(\tau, u) e^{-(t+s-2 \tau)} \\
\quad \times \prod_{i=1}^{d-1} I_{x_{i}}(\gamma(t+s-2 \tau))\left\{I_{u-y}(\gamma(t-\tau))-I_{u+y}(\gamma(t-\tau))\right\} \\
\quad \times\left\{I_{u-y^{\prime}}(\gamma(s-\tau))-I_{u+y^{\prime}}(\gamma(s-\tau))\right\},
\end{aligned}
$$

where the functions $I_{\nu}$ are modified Bessel functions ${ }^{25}$ and where we have taken into account the special form of $b\left(t ; y, y^{\prime}, \vec{x}\right)$ and Eq. (A18). It remains to fix the parameter $\bar{b}(t, y)$, which we determine from the condition $\mathcal{C}(t ; y, y ; \overrightarrow{0})$ $=1$. For large $d$ the factor $\gamma=1 /(T d)$ becomes small, and we can use the following approximation:

$$
I_{y-y^{\prime}}(\gamma(t-\tau)) \approx \delta_{y, y^{\prime}}+O\left(\frac{1}{d}\right)
$$

and similarly for other terms, see also Ref. 24. This yields for vanishing layer magnetization the equation

$$
1=e^{-2 t}+\int_{0}^{t} d \tau e^{-2(t-\tau)} \bar{b}(\tau, y)
$$

for all $y$ and $t$. This equation can be solved by Laplace transform, yielding the result $\bar{b}(t, y)=2$ for all $y$ and $t$. It then follows that the correlation function in the semi-infinite model is given by Eq. (18) with $\bar{b}(t, u)$ set to 2 . One can get rid of the sum over $u$ by using $\sum_{m=-\infty}^{\infty} I_{m+k}\left(z_{1}\right) I_{m}\left(z_{2}\right)=I_{k}\left(z_{1}\right.$ $\left.+z_{2}\right)$ and $I_{n}(z)=I_{-n}(z)$. After doing so, we obtain for the surface autocorrelation function the expression

$$
\begin{aligned}
C_{1}(t, s)= & e^{-(t+s)}\left\{I_{0}(\gamma(t+s))\right\}^{d-1}\left\{I_{0}(\gamma(t+s))-I_{2}(\gamma(t+s))\right\} \\
& +2 \int_{0}^{s} d \tau e^{-(t+s-2 \tau)}\left\{I_{0}(\gamma(t+s-2 \tau))\right\}^{d-1} \\
& \times\left\{I_{0}(\gamma(t+s-\tau))-I_{2}(\gamma(t+s-2 \tau))\right\}
\end{aligned}
$$

It is worth noting that in the limit where $y \approx y^{\prime} \rightarrow \infty$ we also recover the known bulk behavior of the correlation function, as we get with the help of expression (A18) the expression

$$
\begin{aligned}
C\left(t, s ; y, y^{\prime}, \vec{x}\right)= & e^{-(t+s)} I_{y-y^{\prime}}(\gamma(t+s)) \prod_{i=1}^{d-1} I_{x_{i}}(\gamma(t+s)) \\
& +2 \int_{0}^{s} d \tau e^{-(t+s-2 \tau)} \\
& \times I_{y-y^{\prime}}(\gamma(t+s-2 \tau)) \prod_{i=1}^{d-1} I_{x_{i}}(\gamma(t+s-2 \tau))
\end{aligned}
$$

which is precisely the expression found by Garriga et al. ${ }^{24}$ in Fourier space.

We immediately remark that for a quench inside the ordered phase with $T<T_{c}=1$ no simple scaling behavior of the surface autocorrelation is observed, due to the extremely rapidly (i.e., exponentially) increasing Bessel functions. A similar absence of dynamical scaling is also seen in the bulk system quenched below the critical point. ${ }^{24}$ At the critical point, however, when $T=1$ and therefore $\gamma=1 / d$, we can use the approximation $e^{-u} I_{\nu}(u) \approx(2 \pi u)^{-1 / 2} \exp \left[-\nu^{2} /(2 u)\right]$. As the first term in Eq. (21) decreases more rapidly than the second one, we find in the scaling regime (with $Y=t / s$ )

$$
C_{1}(t, s)=4\left(\frac{2 \pi}{d}\right)^{-d / 2} s^{d / 2}\left[(Y-1)^{-d / 2}-(Y+1)^{-d / 2}\right] .
$$

This allows us to identify both the nonequilibrium exponents $b_{1}$ and $\lambda_{C_{1}}$ and the scaling function $f_{C_{1}}(Y)$, see Eq. (6):

$$
b_{1}=\frac{d}{2}, \quad \lambda_{C_{1}}=d+2 \text {, }
$$

$$
f_{C_{1}}(Y)=4\left(\frac{2 \pi}{d}\right)^{-d / 2}\left[(Y-1)^{-d / 2}-(Y+1)^{-d / 2}\right]
$$

where we used that in the limit of large $d$ the critical dynamical exponent is equal to 2 .

\section{B. The response function}

In order to compute the response function we start from the differential equation ${ }^{24}$

$$
\partial_{t}\left\langle\sigma_{\vec{x}, y}(t)\right\rangle=-\left\langle\sigma_{\vec{x}, y}(t)\right\rangle+\left\langle\mathrm{t}_{\vec{x}, y}(t)\right\rangle
$$

for $\left\langle\sigma_{\vec{x}, y}(t)\right\rangle$ in the presence of a small external magnetic field $h_{\vec{x}, y}^{\text {ext }}$. As both $h_{\vec{x}, y}^{\text {ext }}$ and $1 / d$ are small we can develop the tanh to first order in both quantities

$$
\begin{aligned}
\tanh \left[h_{\vec{x}, y}(t) / T\right] \approx & \frac{1}{T} h_{\vec{x}, y}^{\mathrm{ext}}(t)+\frac{\gamma}{2}\left(\sigma_{\vec{x}, y+1}(t)+\sigma_{\vec{x}, y-1}(t)\right. \\
& \left.+\sum_{\vec{z}(\vec{x})} \sigma_{\vec{z}, y}(t)\right)
\end{aligned}
$$

where it is understood that $\sigma_{\vec{x}, 0}=0$. The definition 
TABLE I. Available values of nonequilibrium critical surface quantities at the ordinary transition determined in aging systems quenched to the critical point.

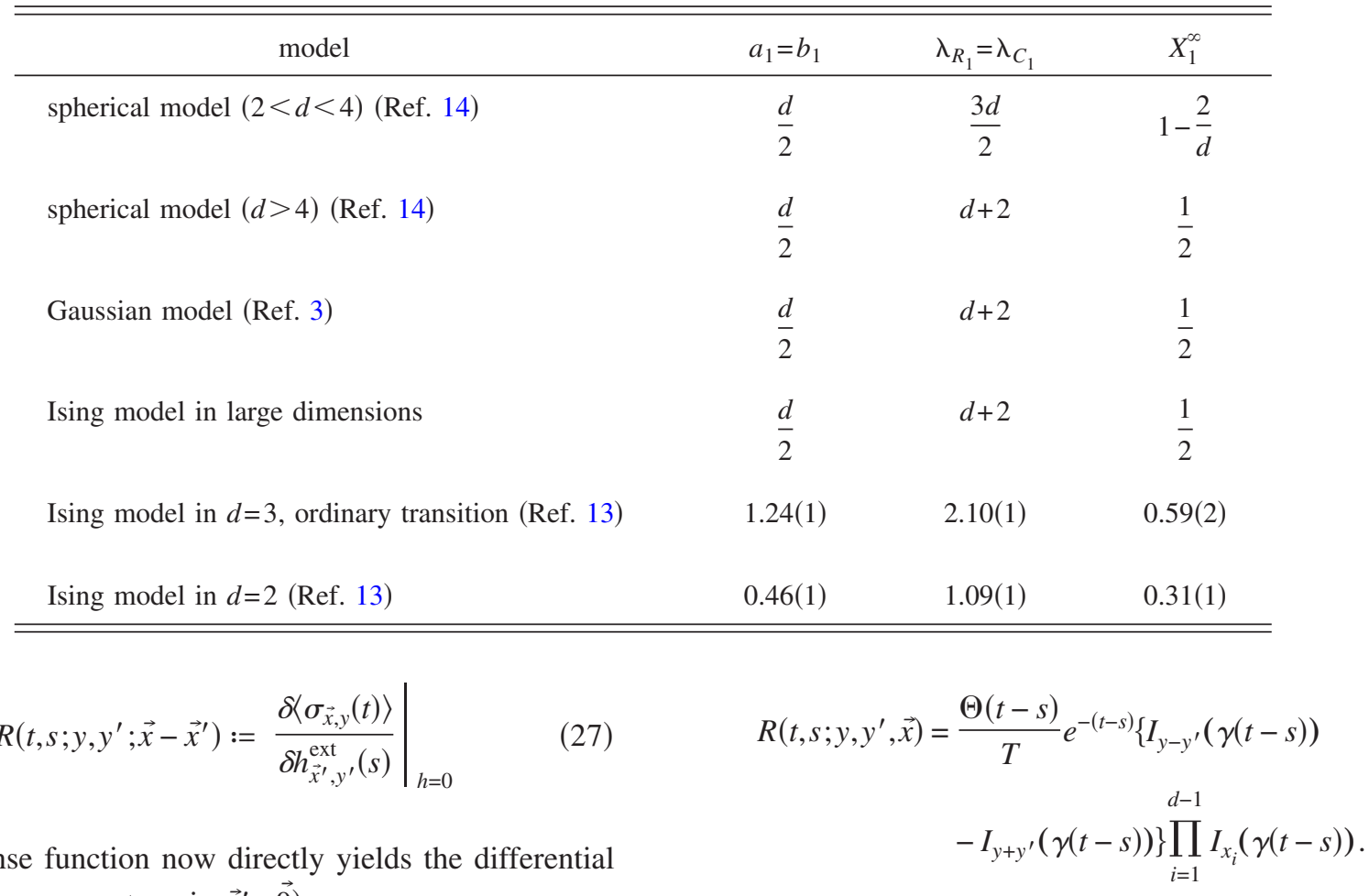

of the response function now directly
equation (where we set again $\vec{x}^{\prime}=\overrightarrow{0}$ )

$$
\begin{aligned}
\partial_{t} R\left(t, s ; y, y^{\prime}, \vec{x}\right)= & -R\left(t, s ; y, y^{\prime}, \vec{x}\right)+\frac{\gamma}{2}\left(R\left(t, s ; y+1, y^{\prime}, \vec{x}\right)\right. \\
& \left.+R\left(t, s ; y-1, y^{\prime}, \vec{x}\right)+\sum_{\vec{z}(\vec{x})} R\left(t, s ; y, y^{\prime}, \vec{z}\right)\right) \\
& +\frac{1}{T} \delta(t-s) \delta_{y, y^{\prime}} \delta_{\vec{x}, \overrightarrow{0}}
\end{aligned}
$$

with $R\left(t, s ; 0, y^{\prime}, \vec{x}\right)=0=R(t, s ; y, 0, \vec{x})$. This equation is solved with similar methods as outlined in the Appendix for the correlation function. As a result we obtain
For the case $T=T_{c}=1$ the surface autoresponse function can again be evaluated in the scaling regime, yielding (with $Y$ $=t / s)$

$$
R_{1}(t, s)=\frac{2 d}{T_{c}}\left(\frac{2 \pi}{d}\right)^{-d / 2} s^{-d / 2-1}(Y-1)^{-(d+2) / 2}
$$

and, therefore,

$$
a_{1}=\frac{d}{2}, \quad \lambda_{R_{1}}=d+2, \quad f_{R_{1}}(Y)=\frac{2 d}{T_{c}}\left(\frac{2 \pi}{d}\right)^{-d / 2}(Y-1)^{-d / 2-1} .
$$

We can now also compute the surface fluctuation-dissipation ratio from the expressions (23) and (30) and obtain

$$
X_{1}(t, s)=\frac{T_{c} R_{1}(t, s)}{\partial_{s} C_{1}(t, s)}=\frac{(Y-1)^{-d / 2-1}}{Y\left[(Y-1)^{-d / 2-1}-(Y+1)^{-d / 2-1}\right]-\left[(Y-1)^{-d / 2}-(Y+1)^{-d / 2}\right]}
$$

from which the limit value $X_{1}^{\infty}=1 / 2$ follows

Comparing with the results obtained for the spherical model, see Table I, we note that the values of the nonequilibrium exponents in the critical short-range Ising model in the limit of high dimensions are in full agreement with the values obtained for the critical spherical model in dimen- sions $d>4 .{ }^{14}$ Even the scaling functions are identical up to a nonuniversal numerical prefactor. In addition, the values of the universal quantities are identical to the values obtained in the field-theoretical Gaussian model. ${ }^{3}$ This nicely demonstrates that in the aging regime universal nonequilibrium features are indeed encountered close to critical surfaces. 


\section{QUENCHING SEMI-INFINITE SYSTEMS FROM HIGH TEMPERATURES: NUMERICAL RESULTS}

In the following we present the results of extensive numerical simulations of the standard two-dimensional semiinfinite Ising model with only nearest-neighbor interactions quenched inside the ordered phase. In the bulk case this model is known to render rather faithfully the physics of real systems undergoing phase ordering. The Hamiltonian is given by the usual expression

$$
\mathcal{H}=-J \sum_{\langle i, j\rangle} \sigma_{i} \sigma_{j}
$$

where $i$ and $j$ label the sites of a semi-infinite lattice. The sum extends over nearest-neighbor pairs, and we have the same coupling strength $J>0$ for every bond connecting neighboring spins. This system exhibits a continuous phase transition at the bulk critical point $T_{c}=2 / \ln (\sqrt{2}+1) \approx 2.269$ (where the temperature is measured in units of $J / k_{B}$, with $k_{B}$ being the Boltzmann constant).

Whereas surface aging behavior has already been studied in the past for Ising models quenched onto the critical point, ${ }^{13}$ this does not seem to be the case for quenches below the critical point. The following numerical study therefore allows us to close a gap in our understanding of the nonequilibrium dynamical behavior of classical spin models. Especially, it yields new insights into the local dynamical behavior of systems undergoing phase ordering in the presence of surfaces.

For these simulations we use periodic boundary conditions in one direction and free boundary conditions in the other direction. We thereby consider square systems with $N$ $=L \times L$ spins were $L$ ranges from $L=300$ to $L=1000$, thus making sure that the data obtained at any one of the two surfaces are representative of the semi-infinite system. Only data free of finite-size effects are discussed in the following. Our focus lies on the surface autocorrelation function and on the surface autoresponse function. The surface autocorrelation function is given by the expression

$$
C_{1}(t, s)=\frac{1}{2 L} \sum_{i \in \text { surface }}\left\langle\sigma_{i}(t) \sigma_{i}(s)\right\rangle,
$$

where the sum is over all the spins in the two surfaces. The data discussed in the following have been obtained after averaging over at least 5000 different runs with different realizations of the noise. In order to study the response to a magnetic field, we apply a weak binary random field between the time $t=0$ (at which the quench takes place) and the time $t=s .{ }^{26}$ After the field has been switched off, we monitor the decay of the surface thermoremanent magnetization given by the expression

$$
M_{1}(t, s)=\frac{1}{2 L} \sum_{i \in \text { surface }} \overline{\left\langle h_{i} \sigma_{i}(t)\right\rangle} / T,
$$

where $h_{i}$ is the strength of the binary random field at site $i$. In addition to averaging over the realizations of the noise we also average over the realizations of the random field as indicated by the bar. We discuss here data obtained with $\left|h_{i}\right|$

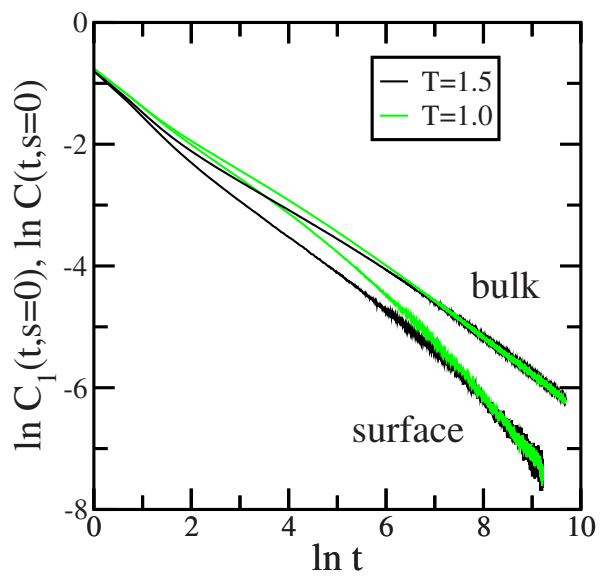

FIG. 1. (Color online) The surface and bulk autocorrelation functions $C_{1}(t, s=0)$ and $C(t, s=0)$ for two different temperatures. At the surface the correlations decay much faster than inside the bulk, yielding the value $\lambda_{C_{1}} / z=0.95$ (3) for the long-time power-law exponent, considerably larger than the value $\lambda_{C} / z=0.63(1)$ in the bulk case.

$=0.1$ (we checked that our conclusions remain the same when we slightly vary the value of $\left|h_{i}\right|$ ). As response functions are very noisy, we average over many more runs than for the autocorrelation. The thermoremanent magnetization data discussed in this section have been obtained after averaging over typically 200000 runs.

\section{A. Autocorrelation function}

Before discussing the surface autocorrelation function, let us briefly mention some results obtained for the autocorrelation function in the corresponding two-dimensional bulk system. The expected scaling form

$$
C(t, s)=s^{-b} f_{C}(t / s) \text { with } f_{C}(t / s) \sim(t / s)^{-\lambda} C^{\prime z} \text { for } t / s \gg 1
$$

has been verified in various numerical studies. These studies showed that $b=0$ and yielded the value $\lambda_{C} / z=0.63(1)$ (Refs. 6,34 , and 35) (recall that $z=2$ ) for the exponent governing the long-time decay of the scaling function. Numerous theoretical approaches have been proposed for computing the scaling function $f_{C},{ }^{27-31}$ the most successful being the recent exploitation of space-time symmetries within the theory of local scale invariance. ${ }^{10,32,33}$

The main question we address here concerns the scaling behavior of the surface autocorrelation function. Let us start by looking at the long-time decay of $C_{1}(t, s)$ with $s=0$, as it is well known that this quantity is usually the most appropriate for the determination of $\lambda_{C_{1}}$. In Fig. 1 we show this quantity for two different temperatures $T=1$ and 1.5 , lower than the critical temperature. For comparison we also include the bulk autocorrelation function $C(t, s=0)$ for the same two temperatures. Whereas at short times the surface autocorrelation (this is also true for the bulk quantity) is clearly temperature dependent, at longer times the two curves get identical. Interestingly, the decay of the surface correlations 

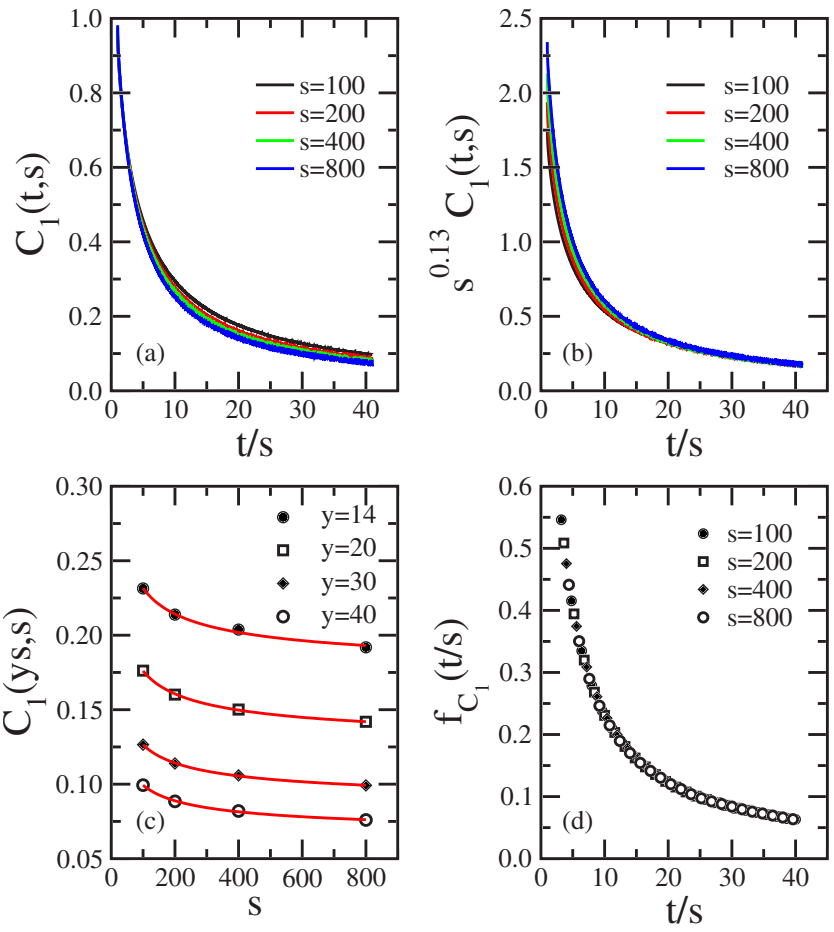

FIG. 2. (Color online) Discussion of the surface autocorrelation $C_{1}(t, s)$ obtained after quenching the semi-infinite two-dimensional Ising model to $T=1$. (a) Autocorrelation as a function of $t / s$ for different values of the waiting time $s$ (the lowest curve shows the data obtained for the largest value of $s$ ). The expected data collapse with $b_{1}=0$ is not observed. (b) Plotting $s^{0.13} C_{1}(t, s)$ versus $t / s$ leads to a collapse of data for large values of $t / s$, but no scaling is observed for smaller values of $t / s$. (c) Plot of $C_{1}(y s, s)$ as a function of $s$ for various values of $y=t / s$. The full lines are fits to the extended scaling form (37) with $b^{\prime}=0.49$. (d) Scaling function $f_{C_{1}}(t / s)$ obtained from the data shown in (a) after subtracting off the finite-time correction term. In (c) and (d) error bars are smaller than the symbol sizes.

follow a power-law at late times. This power-law decay is faster at the surface than inside the bulk, yielding the value $\lambda_{C_{1}} / z=0.95(3)$ which should be compared to the value $\lambda_{C} / z=0.63$ (1) obtained inside the bulk. Obviously, this faster decay is due to the reduced coordination number at the surface.

In Fig. 2 we discuss the behavior of the surface autocorrelation function $C_{1}(t, s)$ with $s>0$. When plotting $C_{1}(t, s)$ versus $t / s$, we do not observe a data collapse, see Fig. 2(a), in contrast to the data collapse observed when plotting the bulk autocorrelation as a function of $t / s$. The data shown in Fig. 2(a) at first look suggest that the local exponent $b_{1}$ is different from zero at the surface. A more thorough analysis reveals however that a good scaling behavior can not be achieved with a constant $b_{1}>0$. Figure 2(b) shows our best result obtained for $b_{1}=0.13$. A reasonable data collapse can be achieved this way for large values of $t / s$, but scaling breaks down for $t / s \leqslant 25$. Taken at face value, this would suggest for the surface autocorrelation function the existence of a large threshold value of $t / s$ below which dynamical scaling is not observed. The possible physical mechanism responsible for this threshold is far from obvious. A better data collapse can be achieved by allowing the exponent $b_{1}$ to depend itself on $t / s$, but a nonconstant exponent varying as a function of $t / s$ is not supported by any theoretical approach.

We propose here another interpretation of the numerical data that is based on the recent observation that large finitetime corrections can to some extend mask the true scaling behavior of the autocorrelation function in phase-ordering systems. ${ }^{4}$ In order to take the existence of finite-time corrections into account, we try to describe our data by the ansatz

$$
C_{1}(t, s)=f_{C_{1}}(t / s)+s^{-b^{\prime}} g_{C_{1}}(t / s),
$$

where the first term is the expected scaling behavior with $b_{1}=0$, whereas the second term is the finite-time correction that is of decreasing importance for increasing values of the waiting time $s$. This ansatz has recently been used for the analysis of the autocorrelation functions in disordered ferromagnets quenched below their critical point. ${ }^{4,36}$ In Fig. 2(c) we show $C_{1}(y s, s)$ as a function of $s$ for various values of the ratio $y=t / s$. The lines show that an excellent fitting of the data can be achieved with the extended scaling form (37) with a common value $b^{\prime}=0.49(1)$. The scaling function $f_{C_{1}}(t / s)$, obtained after subtracting off the correction term, is shown in Fig. 2(d). As the curves for the different values of $s$ are not distinguishable on the scale of the figure, we only show selected points as symbols. The data collapse shown in Fig. 2(d) supports our interpretation that the true scaling behavior of the surface autocorrelation function is masked by strong finite-time corrections. As a consistency check, we note that the data in Fig. 2(d) present for large values of $t / s$ a power-law decay with an exponent $0.95(2)$, in full agreement with the value of $\lambda_{C_{1}} / z$ obtained directly from $C_{1}(t, s$ $=0)$. Even though our data are perfectly described by Eq. (37), we must emphasize that we do not yet know why this finite-time correction shows up close to the surface but is not encountered inside the bulk.

Let us end the discussion of the surface autocorrelation function by noticing that the value $b^{\prime}=0.49(1)$ of the correction term exponent is compatible with $1 / 2=1 / z$. However, we refrain from making the conjecture $b^{\prime}=1 / z$ here without having studied other systems with surfaces (such as, for example, semi-infinite Potts models).

\section{B. Response function}

Before discussing the surface thermoremanent magnetization $M_{1}(t, s)$, let us again first recall the behavior of the corresponding bulk quantity. The bulk thermoremanent magnetization $M(t, s)$ is a temporally integrated response function that is related to the response function $R(t, s)$ by the integral

$$
M(t, s)=\int_{0}^{s} d u R(t, u)
$$

where the integration is over the whole time interval during which the magnetic field was acting on the system. From the scaling form (4) of $R(t, s)$, we therefore obtain the scaling behavior 

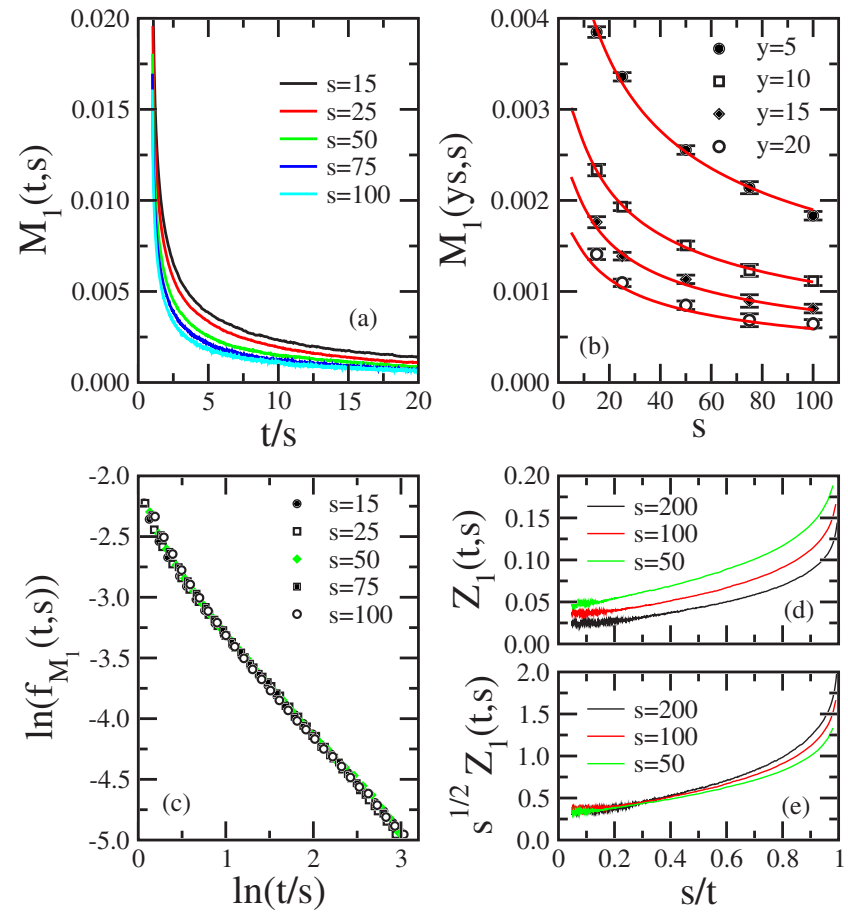

FIG. 3. (Color online) Discussion of the surface thermoremanent magnetization $M_{1}(t, s)$ obtained after quenching the semiinfinite two-dimensional Ising model to $T=1$, where a random magnetic field of strength $h=0.1$ is applied between $t=0$ and $t=s$. (a) $M_{1}(t, s)$ plotted against $t / s$ for various waiting times $s$ (the lowest curve shows the data obtained for the largest value of $s$ ). (b) Plot of $M_{1}(y s, s)$ as a function of $s$ for various values of $y=t / s$. The full lines are fits to the extended scaling form (42). A consistent description of the data for any value of $t / s$ is achieved for $\lambda_{R} / z=0.95, a$ $=0.5$, and $r_{1}=-0.106$, see main text. (c) Scaling function $f_{M_{1}}(t / s)$ obtained from the data shown in (a) after subtracting off the finitetime correction term. Error bars are smaller than the symbol sizes. (d) Plot of $Z_{1}(t, s)$, see Eq. (43), versus $s / t$ for different waiting times. (e) Plotting $s^{1 / 2} Z_{1}(t, s)$ versus $s / t$ leads to a data collapse for small values of $s / t$.

$$
M(t, s)=s^{-a} f_{M}(t / s)
$$

for the integrated response. Zippold, Kühn, and Horner ${ }^{37}$ were the first to point out the existence of a subleading correction term which can be quite sizeable. For the thermoremanent magnetization this leads to the following more complete scaling behavior: ${ }^{9}$

$$
M(t, s)=s^{-a} f_{M}(t / s)+s^{-\lambda_{R} / z} g_{M}(t / s) .
$$

The second term in this equation is in fact the response of the system to fluctuations in the initial state, where the scaling function $g_{M}(t / s)$ is expected to be proportional to the power law $(t / s)^{-\lambda_{R} / z}{ }^{38}$ For the two-dimensional Ising model we have $a=1 / z=1 / 2$ and $\lambda_{R} / z=0.63$. Therefore this correction to scaling can not be neglected but must be included in order to obtain the correct description of the scaling behavior of the bulk thermoremanent magnetization. ${ }^{9,10}$

In Fig. 3 we summarize our findings for the surface thermoremanent magnetization in the two-dimensional semiinfinite Ising model quenched below the critical point. Figure 3(a) shows the behavior of this local response as a function of $t / s$ for various values of the waiting time $s$. In a first attempt, we might try to achieve a scaling behavior by assuming that

$$
M_{1}(t, s)=s^{-a_{1}} f_{M_{1}}(t / s),
$$

thereby neglecting any possible corrections to scaling. A reasonable scaling behavior is achieved this way for a value of $a_{1} \approx 0.40$, slightly lower than the expected value $1 / z=1 / 2$. For a more thorough analysis we can fix $y=t / s$ and plot the response as a function of the waiting time in a log-log plot. Fitting a straight line to the data, we obtain from the slope of that line a value of $a_{1}$ for every considered value of $t / s$. Thus, we obtain $a_{1}=0.38$ for $t / s=5, a_{1}=0.39$ for $t / s=10$, $a_{1}=0.40$ for $t / s=15$, and $a_{1}=0.42$ for $t / s=20$. This points to the existence of a correction term that vanishes for increasing values of $t / s$. In Fig. 3(b) we test the more complete scaling form

$$
M_{1}(t, s)=s^{-a_{1}} f_{M_{1}}(t / s)+s^{-\lambda_{R_{1}} / z} g_{M_{1}}(t / s),
$$

where the correction term with the scaling function $g_{M_{1}}(t / s)=r_{1}(t / s)^{-\lambda_{R_{1}} / z}$ describes the response of the surface to fluctuations in the initial state. Plugging in the value $\lambda_{R_{1}} / z=0.95$ (where we assume that $\lambda_{R_{1}}=\lambda_{C_{1}}$ holds), we obtain a consistent description for any $t / s$ with common values $r_{1}=-0.106(1)$ for the amplitude of the correction term and $a_{1}=0.50$ (1) for the exponent of the leading term. The correction term being now completely fixed, we can subtract it off from the numerical data and obtain the data collapse shown in Fig. 3(c). Thus, as for the thermoremanent magnetization in the bulk, $, 9,10$ we are able to identify the leading correction term and, in addition, obtain the value $a_{1}=a=1 / z$.

We close this section by a brief discussion of the surface fluctuation-dissipation ratio. In Fig. 3(d) we plot the ratio

$$
Z_{1}(t, s)=\frac{T M_{1}(t, s)}{h C_{1}(t, s)}
$$

as a function of $s / t$ for various values of $s$. This ratio yields asymptotically the limit value $X_{1}^{\infty}$ of the fluctuationdissipation ratio $(8)$, as

$$
X_{1}^{\infty}=\lim _{s \rightarrow \infty}\left[\lim Z_{1 \rightarrow \infty}(t, s)\right] .
$$

For a given value of the waiting time, the ratio $Z_{1}(t, s)$ converges towards a constant finite value when $s / t \rightarrow 0$. At first look this might seem surprising as in coarsening systems one expects the limit value $X_{1}^{\infty}=0$. However, this constant decreases for increasing values of $s$. Taking into consideration the leading scaling behaviors of $C_{1}(t, s) \sim f_{C_{1}}(t / s)$ and of $M_{1}(t, s) \sim s^{-1 / 2} f_{M_{1}}(t / s)$ found in our study as well as the fact that the scaling functions $f_{C_{1}}(t / s)$ and $f_{M_{1}}(t / s)$ display for large arguments a power-law behavior with the same exponent 0.95 , we find that the saturation value $\lim _{t \rightarrow \infty} Z_{1}(t, s)$ should vanish as $s^{-1 / 2}$. This is indeed verified in Fig. 3(e), where $s^{1 / 2} Z_{1}(t, s)$ leads to a collapse of the data onto a common curve for $s / t$ small. This is also an a posteriori check that we have indeed correctly identified the leading scaling 
behaviors of both the surface autocorrelation and the surface integrated response functions.

\section{CONCLUSIONS}

In this paper we have extended the investigation of surface aging phenomena to cases not studied previously. On the one hand, we have computed nonequilibrium surface quantities in the exactly solvable short-range semi-infinite Ising model in the limit of a large number of space dimensions. On the other hand, we have presented numerical simulations of the standard semi-infinite Ising model quenched inside the ordered phase.

For a quench to the critical point, we added the semiinfinite Ising model in high dimensions to the list of exactly solved models. The universal nonequilibrium surface quantities obtained in this study agree with those obtained for the critical semi-infinite spherical model, ${ }^{14}$ as expected for a mean-field-like model. In Table I we summarize the known results for surface aging phenomena in critical systems at the ordinary transition (the only situation studied in this paper) by listing the values of the different universal nonequilibrium exponents as well as those of the asymptotic value of the fluctuation-dissipation ratio. It is worth mentioning that the existing numerical data for the semi-infinite Ising model ${ }^{13}$ indicate a nonmonotonic behavior of the limit value of the surface fluctuation-dissipation ratio as a function of the dimensionality of the system (being $\frac{1}{2}$ for $d \geqslant 4$, then increasing to 0.59 in three dimensions, before decreasing to 0.31 in the two-dimensional system). This behavior is unexpected, and a satisfactory explanation is still lacking.

We also presented large-scale numerical simulations of the two-dimensional semi-infinite Ising model undergoing coarsening. From these results we conclude that the result $b_{1} \neq 0$ found for the spherical mode ${ }^{14}$ is not generic but that it is very probably an artifact of that rather artificial model. Indeed, the numerical simulations of the more realistic twodimensional Ising model yield $b_{1}=0$. This indicates that generically the exponent $b_{1}$, that governs the scaling of the surface correlations, vanishes, similarly to what is observed inside the bulk.

One of the main conclusions of our work is that surface aging phenomena in systems undergoing phase ordering display the same general features as bulk aging phenomena. Simple scaling forms prevail asymptotically for two-time quantities such as the surface autoresponse and the surface autocorrelation functions, and universal nonequilibrium quantities, with values that differ from the bulk values, can also be identified in semi-infinite coarsening systems, see Table II. For finite times, corrections to scaling can be rather important and might even mask the leading scaling behavior. In our study of the two-dimensional semi-infinite Ising model we not only identified a subleading contribution to the thermoremanent surface magnetization (a similar correction also appears inside the bulk), but we also showed the existence of corrections to scaling in the surface autocorrelation function. The physical origin of this last term is not yet clear. It is, however, worth noting that a similar correction term has recently been shown to exist for the random bond Ising model quenched below the critical point. ${ }^{4}$
TABLE II. Available values of nonequilibrium surface quantities determined in aging systems quenched below the critical point.

\begin{tabular}{cccc}
\hline \hline model & $a_{1}$ & $b_{1}$ & $\lambda_{R_{1}}=\lambda_{C_{1}}$ \\
\hline spherical model (Ref. 14) & $\frac{d}{2}$ & 1 & $\frac{d}{2}+2$ \\
Ising model in $d=2$ & $\frac{1}{2}$ & 0 & $1.90(6)$ \\
& & & \\
\hline \hline
\end{tabular}

The semi-infinite geometry discussed in this paper is of course only a special case of a more general wedge-shaped geometry. Wedges in critical systems have been studied quite intensively in the past, ${ }^{18,39-41}$ as they lead to static critical quantities whose values depend on the opening angle of the wedge. However, the local critical dynamical behavior in a wedge-shaped geometry has not yet been discussed in the literature. Phase ordering in wedges can also be viewed as being one of the simplest cases of phase-ordering in confined geometries. The study of edge aging phenomena is therefore the next logical step in the study of local nonequilibrium dynamical behavior in confined geometries, and work along this line is in progress.

\section{ACKNOWLEDGMENTS}

We acknowledge the support of the Deutsche Forschungsgemeinschaft through Grant No. PL 323/2 and by the Franco-German binational programme PROCOPE. The numerical work was done on Virginia Tech's System X.

\section{APPENDIX: ISING MODEL IN HIGH DIMENSIONS: COMPUTATION OF THE CORRELATION FUNCTION}

In this Appendix we compute the one-time and two-times correlation functions for the semi-infinite Ising model in high dimensions. We thereby start by defining the operator $\square_{t, y, y^{\prime}, \vec{x}}^{(\gamma)}$ for a function $f: \mathbb{R} \times \mathbb{Z}_{\geqslant 0} \times \mathbb{Z}_{\geqslant 0} \times \mathbb{Z}^{d-1} \rightarrow \mathbb{R}$ :

$$
\begin{aligned}
\square_{t ; y, y^{\prime}, \vec{x}}^{(\gamma)} f\left(t ; y, y^{\prime}, \vec{x}\right):= & \partial_{t} f\left(t ; y, y^{\prime}, \vec{x}\right)+2 f\left(t ; y, y^{\prime}, \vec{x}\right) \\
& -\frac{\gamma}{2}\left(f\left(t ; y+1, y^{\prime}, \vec{x}\right)+f\left(t ; y-1, y^{\prime}, \vec{x}\right)\right. \\
& +f\left(t ; y, y^{\prime}+1, \vec{x}\right)+f\left(t ; y, y^{\prime}-1, \vec{x}\right) \\
& \left.+2 \sum_{\vec{x}^{\prime}(\vec{x})} f\left(t ; y, y^{\prime}, \vec{x}^{\prime}\right)\right)
\end{aligned}
$$

where $\vec{x}^{\prime}(\vec{x})$ denotes the nearest neighbors of $\vec{x}$ in the layer $y$. With this, Eq. (14) reads

$$
\square_{t ; y, y^{\prime}, \vec{x}}^{(\gamma)} C\left(t ; y, y^{\prime}, \vec{x}\right)=b\left(t ; y, y^{\prime}, \vec{x}\right) .
$$

In order to solve this equation we look for the Green's function satisfying the equation

$$
\square_{t ; y, y^{\prime}, \vec{x}}^{(\gamma)} g\left(t ; u, y, v, y^{\prime}, \vec{x}\right)=\delta(t) \delta_{y, u} \delta_{y^{\prime}, v} \delta_{\vec{x}, 0}
$$


and the boundary conditions

$$
g\left(t ; u, 0, v, y^{\prime}, \vec{x}\right)=0=g(t ; u, y, v, 0, \vec{x}) .
$$

We can solve this equation by using a Fourier-Sine transformation on $y$ and $y^{\prime}$ (which makes sure the boundary conditions hold) and a normal Fourier transformation on $\vec{x}$ :

$$
\begin{aligned}
\hat{g}\left(t ; u, k, v, k^{\prime}, \vec{q}\right)= & \sum_{y, y^{\prime} \geqslant 0} \sum_{\vec{x}} \sin (k y) \sin \left(k^{\prime} y^{\prime}\right) \\
& \times e^{i \vec{x} \cdot \vec{q}} g\left(t ; u, y, v, y^{\prime}, \vec{x}\right), \\
g\left(t ; u, y, v, y^{\prime}, \vec{x}\right)= & \int_{0}^{\pi} \frac{d k}{\pi / 2} \int_{0}^{\pi} \frac{d k^{\prime}}{\pi / 2} \int_{\mathcal{B}} \frac{d \vec{q}}{(2 \pi)^{d-1}} \\
& \times \sin (k y) \sin \left(k^{\prime} y^{\prime}\right) e^{-i \vec{x} \cdot \vec{q}} \hat{g}\left(t ; u, k, v, k^{\prime}, \vec{q}\right) .
\end{aligned}
$$

Here, the sums are over all lattice sites, whereas $\mathcal{B}=[-\pi, \pi]^{d-1}$ is the first Brillouin zone and $d \vec{q}=\prod_{i=1}^{d-1} d q_{i}$. It is straightforward to work out Eq. (A3) in Fourier space, which yields

$$
\begin{aligned}
& \partial_{t} \hat{g}\left(t ; u, k, v, k^{\prime}, \vec{q}\right)+\omega\left(k, k^{\prime}, \vec{q}\right) \hat{g}\left(t ; u, k, v, k^{\prime}, \vec{q} ; t\right) \\
& \quad=\sin (u k) \sin \left(v k^{\prime}\right) \delta(t),
\end{aligned}
$$

where the expression $\omega\left(k, k^{\prime}, \vec{q}\right)$ is given by

$$
\omega\left(k, k^{\prime}, \vec{q}\right)=\omega(k)+\omega\left(k^{\prime}\right)+\omega(\vec{q})
$$

with

$$
\begin{gathered}
\omega(k)=\left(\frac{1}{d}-\gamma \cos (k)\right), \\
\omega\left(k^{\prime}\right)=\left(\frac{1}{d}-\gamma \cos \left(k^{\prime}\right)\right), \\
\omega(\vec{q})=\sum_{i=1}^{d-1}\left(\frac{2}{d}-2 \gamma \cos \left(q_{i}\right)\right) .
\end{gathered}
$$

Equation (A7) is readily solved and yields the result

$$
\hat{g}\left(t ; u, k, v, k^{\prime}, \vec{r} ; t\right)=\Theta(t) \sin (u k) \sin \left(v k^{\prime}\right) \exp \left[-\omega\left(k, k^{\prime}, \vec{q}\right) t\right],
$$

where $\Theta(t)$ is the Heaviside step function. This expression still has to be brought back to direct space using Eq. (A6). With the integral $\int_{-\pi}^{\pi} d k \exp [i r k+\cos (k) z]=2 \pi I_{r}(z)$ this yields the result

$$
\begin{aligned}
g\left(t ; u, y, v, y^{\prime}, \vec{x}\right)= & \Theta(t) e^{-2 t}\left[I_{u-y}(\gamma t)-I_{u+y}(\gamma t)\right]\left[I_{v-y^{\prime}}(\gamma t)\right. \\
& \left.-I_{v+y^{\prime}}(\gamma t)\right] \prod_{i=1}^{d-1} I_{x_{i}}(2 \gamma t) .
\end{aligned}
$$

With the help of this function, the inhomogeneous differential equation (14) is solved by

$$
\begin{aligned}
\mathcal{C}\left(t ; y, y^{\prime}, \vec{x}\right)= & \mathcal{C}_{h}\left(t ; y, y^{\prime}, \vec{x}\right) \\
& +\sum_{u, v \geqslant 0} \sum_{\vec{x}^{\prime}} \int_{0}^{\infty} d \tau g\left(t-\tau ; u, y, v, y^{\prime}, \vec{x}-\vec{x}\right) \\
& \times b\left(\tau ; u, v, \vec{x}^{\prime}\right),
\end{aligned}
$$

where $\mathcal{C}_{h}\left(t ; y, y^{\prime}, \vec{x}\right)$ is an arbitrary solution of the homogeneous equation $\square_{t ; y, y^{\prime}, \mathcal{C}_{h}}^{(\gamma)}\left(t ; y, y^{\prime}, \vec{x}\right)=0$ that satisfies the boundary condition (16).

In order to obtain this special solution $\mathcal{C}_{h}\left(t ; y, y^{\prime}, \vec{x}\right)$ we start from Eq. (A3) with a vanishing right-hand side

$$
\square_{t ; y, y^{\prime},{ }_{x}} \mathcal{C}_{h}\left(t ; y, y^{\prime}, \vec{x}\right)=0
$$

for which we obtain the solution

$$
\hat{\mathcal{C}}_{h}\left(t ; k, k^{\prime}, \vec{q}\right)=\exp \left[-\omega\left(k, k^{\prime}, \vec{q}\right) t\right] \hat{\mathcal{C}}_{h}\left(0 ; k, k^{\prime}, \vec{q}\right)
$$

in Fourier space, where the initial value $\hat{\mathcal{C}}_{h}\left(0 ; k, k^{\prime}, \vec{q}\right)$ is given by

$$
\hat{\mathcal{C}}_{h}\left(0 ; k, k^{\prime}, \vec{q}\right)=\sum_{u, v \geqslant 0} \sum_{\vec{x}^{\prime}(\vec{x})} \sin (u k) \sin \left(v k^{\prime}\right) e^{-\mathrm{i} \vec{x}^{\prime} \vec{q}} \mathcal{C}\left(0 ; u, v, \vec{x}^{\prime}\right)
$$

Equation (15) for the two-time correlator is also solved in a similar way. We go to Fourier space and get the solution

$$
\hat{C}\left(t, s ; k, k^{\prime}, \vec{q}\right)=\exp \left(-\frac{\gamma}{2}[\omega(k)+\omega(\vec{q})](t-s)\right) \hat{\mathcal{C}}\left(s ; k, k^{\prime}, \vec{q}\right)
$$

Inserting the Fourier transform of the solution of Eq. (A12), we obtain after transforming back to real space the final result 


$$
\begin{aligned}
C\left(t, s ; y, y^{\prime}, \vec{x}\right)= & \sum_{u, v \geqslant 0} \sum_{\vec{x}^{\prime}(\vec{x})} \mathcal{C}\left(0 ; u, v, \vec{x}^{\prime}\right) e^{-(t+s)} \prod_{i=1}^{d-1} I_{r_{i}-r_{i}^{\prime}}(\gamma(t+s))\left[I_{u-y}(\gamma t)-I_{u+y}(\gamma t)\right]\left[I_{v-y^{\prime}}(\gamma s)-I_{v+y^{\prime}}(\gamma s)\right] \\
& +\sum_{u, v \geqslant 0} \sum_{\vec{x}^{\prime}(\vec{x})} \int_{0}^{\infty} d \tau b\left(\tau ; u, v, \vec{x}^{\prime}\right) e^{-(t+s-2 \tau)} \\
& \times \prod_{i=1}^{d-1} I_{x_{i}-x_{i}^{\prime}}(\gamma(t+s-2 \tau))\left\{I_{u-y}(\gamma(t-\tau))-I_{u+y}(\gamma(t-\tau))\right\}\left\{I_{u-y}(\gamma(s-\tau))-I_{u+y}(\gamma(s-\tau))\right\} .
\end{aligned}
$$

For decorrelated initial conditions $\mathcal{C}\left(0 ; u, v, \vec{x}^{\prime}\right)=\delta_{u, v} \delta_{\vec{x}^{\prime}, 0}$, one can rearrange the Bessel functions in the first sum using $\sum_{\nu=-\infty}^{\infty} I_{\nu}\left(z_{1}\right) I_{\nu+k}\left(z_{2}\right)=I_{k}\left(z_{1}+z_{2}\right)$ which gives

$$
\sum_{u \geqslant 0}\left[I_{u-y}(\gamma t)-I_{u+y}(\gamma t)\right]\left[I_{u-y^{\prime}}(\gamma s)-I_{u+y^{\prime}}(\gamma s)\right]=I_{y-y^{\prime}}(\gamma(t+s))-I_{y+y^{\prime}}(\gamma(t+s)),
$$

an equation needed for deriving the final result (21).

${ }^{1}$ A. J. Bray, Adv. Phys. 43, 357 (1994).

${ }^{2}$ L. F. Cugliandolo, in Slow Relaxation and Non Equilibrium Dynamics in Condensed Matter, edited by J.-L. Barrat, J. Dalibard, J. Kurchan, and M. V. Feigel'man (Springer, Berlin, 2003).

${ }^{3}$ P. Calabrese and A. Gambassi, J. Phys. A 38, R133 (2004).

${ }^{4}$ M. Henkel and M. Pleimling, in Rugged Free Energy Landscapes: Common Computational Approaches in Spin Glasses, Structural Glasses and Biological Macromolecules, edited by W. Janke (Springer, Heiselberg, in press).

${ }^{5}$ Ageing and the Glass Transition, edited by M. Henkel, M. Pleimling, and R. Sanctuary, Springer Lecture Notes in Physics No. 716 (Springer, Heidelberg, 2007).

${ }^{6}$ D. S. Fisher and D. A. Huse, Phys. Rev. B 38, 373 (1988).

${ }^{7}$ D. A. Huse, Phys. Rev. B 40, 304 (1989).

${ }^{8}$ A. Picone and M. Henkel, J. Phys. A 35, 5575 (2002).

${ }^{9}$ M. Henkel, M. Paessens, and M. Pleimling, Europhys. Lett. 62, 644 (2003); Phys. Rev. E 69, 056109 (2004).

${ }^{10}$ E. Lorenz and W. Janke, Europhys. Lett. 77, 10003 (2007).

${ }^{11}$ H. K. Janssen, B. Schaub, and B. Schmittmann, Z. Phys. B: Condens. Matter 73, 539 (1989).

${ }^{12}$ A. Picone and M. Henkel, Nucl. Phys. B 688, 217 (2004).

${ }^{13}$ M. Pleimling, Phys. Rev. B 70, 104401 (2004).

${ }^{14}$ F. Baumann and M. Pleimling, J. Phys. A 39, 1981 (2006).

${ }^{15} \mathrm{~K}$. Binder, in Phase Transitions and Critical Phenomena (Academic, London, 1983), Vol. 8.

${ }^{16}$ H. W. Diehl, in Phase Transitions and Critical Phenomena (Academic, London, 1986), Vol. 10.

${ }^{17}$ H. W. Diehl, Int. J. Mod. Phys. B 11, 3503 (1997).

${ }^{18}$ M. Pleimling, J. Phys. A 37, R79 (2004).

${ }^{19}$ S. Dietrich and H. W. Diehl, Z. Phys. B: Condens. Matter 51, 343 (1983).

${ }^{20}$ U. Ritschel and P. Czerner, Phys. Rev. Lett. 75, 3882 (1995).
${ }^{21}$ S. N. Majumdar and A. M. Sengupta, Phys. Rev. Lett. 76, 2394 (1996).

${ }^{22}$ C. Godrèche and J.-M. Luck, J. Phys.: Condens. Matter 14, 1589 (2002).

${ }^{23}$ A. Crisanti and F. Ritort, J. Phys. A 36, R181 (2003).

${ }^{24}$ A. Garriga, P. Sollich, I. Pagonabarraga, and F. Ritort, Phys. Rev. E 72, 056114 (2005).

${ }^{25}$ I. S. Gradshteyn and I. M. Ryzhik, Table of Integrals, Series, and Products, 6th ed. (Academic, London, 1980).

${ }^{26}$ A. Barrat, Phys. Rev. E 57, 3629 (1998).

${ }^{27}$ A. J. Bray and S. Puri, Phys. Rev. Lett. 67, 2670 (1991); H. Toyoki, Phys. Rev. B 45, 1965 (1992).

${ }^{28}$ A. J. Bray and K. Humayun, Phys. Rev. E 48, R1609 (1993).

${ }^{29}$ F. Liu and G. F. Mazenko, Phys. Rev. B 44, 9185 (1991).

${ }^{30}$ F. Rojas and A. D. Rutenberg, Phys. Rev. E 60, 212 (1999).

${ }^{31}$ G. F. Mazenko, Phys. Rev. E 58, 1543 (1998).

${ }^{32}$ M. Henkel, A. Picone, and M. Pleimling, Europhys. Lett. 68, 191 (2004).

${ }^{33}$ M. Henkel and F. Baumann, J. Stat. Mech.: Theory Exp. (2007) P07015.

${ }^{34}$ G. Brown, P. A. Rikvold, M. Sutton, and M. Grant, Phys. Rev. E 56, 6601 (1997).

${ }^{35}$ M. Henkel and M. Pleimling, Phys. Rev. E 68, 065101(R) (2003).

${ }^{36}$ M. Henkel and M. Pleimling, Europhys. Lett. 76, 561 (2006).

${ }^{37}$ W. Zippold, R. Kühn, and H. Horner, Eur. Phys. J. B 13, 531 (2000).

${ }^{38}$ K. Humayun and A. J. Bray, J. Phys. A 24, 1915 (1991).

${ }^{39}$ J. Cardy, J. Phys. A 16, 3617 (1983).

${ }^{40}$ F. Iglói, I. Peschel, and L. Turban, Adv. Phys. 42, 683 (1993).

${ }^{41}$ M. Pleimling and W. Selke, Eur. Phys. J. B 5, 805 (1998). 